Résumés des conférences et travaux

\title{
Archéologie grecque
}

\section{Application des moyens informatiques aux sciences historiques}

Conception d'une base de données sur la sculpture antique en rapport avec des exemples d'autres bases de données

\section{Marie-Françoise Clergeau}

\section{OpenEdition}

\section{Journals}

Édition électronique

URL : https://journals.openedition.org/ashp/204

DOI : 10.4000/ashp.204

ISSN : 1969-6310

\section{Éditeur}

Publications de l'École Pratique des Hautes Études

Édition imprimée

Date de publication : 1 octobre 2008

Pagination : 78-83

ISSN : 0766-0677

Référence électronique

Marie-Françoise Clergeau, "Application des moyens informatiques aux sciences historiques », Annuaire de l'École pratique des hautes études (EPHE), Section des sciences historiques et philologiques [En ligne], 139 | 2008, mis en ligne le 24 novembre 2008, consulté le 12 juillet 2021. URL : http:// journals.openedition.org/ashp/204; DOI : https://doi.org/10.4000/ashp.204 


\title{
APPLICATION DES MOYENS INFORMATIQUES AUX SCIENCES HISTORIQUES
}

\author{
Chargée de conférences : $\mathrm{M}^{\mathrm{me}}$ Marie-Françoise ClergeaU
}

Programme de l'année 2006-2007 : Conception d'une base de données sur la sculpture antique en rapport avec des exemples d'autres bases de données.

Nous avons mené une réflexion méthodologique sur la constitution d'une base de données dans un domaine bien défini de l'archéologie figurée concernant la statuaire grecque et romaine, au sein de l'équipe d'accueil « Histoire de l'art, histoire des représentations et archéologie de l'Europe : sources, documents et méthodes », dirigée par M. François Queyrel (EA 4115).

\section{Délimitation du sujet et méthode}

Loin de viser à développer une base encyclopédique qui puise à toutes les sources concernant la statuaire grecque et romaine, le but est de rendre scientifiquement plus accessible, grâce à l'informatique, le corpus de données du Répertoire de la statuaire grecque et romaine de Salomon Reinach (conservateur des musées nationaux, Membre

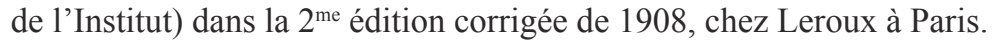

D'emblée, nous avons écarté l'idée de faire une nouvelle publication en ligne et d'y greffer un outil de recherche en plein texte. Notre expérience des projets informatiques dans le domaine de l'histoire de l'art nous a appris que ces solutions procurent au « grand public » une interrogation aisée mais qu'elles sont inadaptées dès lors que des spécialistes doivent obtenir de façon exhaustive les objets de leur recherche. Nous avons donc opté pour une information structurée en base de données, et cela d'autant plus volontiers que la démarche de Salomon Reinach en constituait la prémisse exemplaire.

Une des remarques de l'auteur, dans l'introduction à son ouvrage, mériterait d'être enseignée à tous les responsables des bases de données :

«... il s'agit, en effet, non pas de publier, mais de préparer un corpus statuarum, l'essentiel n'est-il pas d'abord de classer les documents, de constater leur existence, de fixer l'individualité de chacun par une image? »

De la même façon, les bases de données sont des outils qui doivent demeurer « objectifs » et non pas des livres d'étude où l'auteur avance ses hypothèses, donne le résultat de ses réflexions. Aussi avons-nous cherché à demeurer fidèles aux données de l'ouvrage, excluant toute structure ou terminologie qui pût introduire une part d'interprétation. L'objectivité passe par l'abandon de réflexes de pensée et d'expression inhérents à notre époque, c'est pourquoi nous avons jugé nécessaire de consacrer les premières conférences à l'étude historique de l'auteur. Ainsi avons-nous pu apprécier 
le recul - voire l'humour - de Salomon Reinach par rapport à l'objet de ses recherches et, dans sa démarche de classement, le bon-sens dominant l'esprit de système, cet esprit de système que les nouvelles technologies peuvent à notre insu induire dans notre mode de pensée. Une incursion dans « l'affaire » Glozel et dans la bibliographie de l'auteur donne un éclairage non seulement sur la culture que chacun lui reconnaît, mais encore sur l'élégance de son esprit et la largeur de ses vues.

Fruit de cinq années de recherche, la compilation de Salomon Reinach propose un matériau de plusieurs milliers de croquis au trait, assortis de très courtes notices d'informations bibliographiques et muséographiques. Un appareil typologique, bibliographique et un « ordre des matières " permettent de compléter ces notices abrégées. Le recensement couvre les collections et les publications sur le champ international (jusqu'en 1908), et par là, présente des points communs avec le Musée virtuel achéménide (MAVI), à la conception duquel nous participons sous la direction du professeur Pierre Briant au Collège de France. Mais il en diffère essentiellement par son éloignement du concret et le fini de son contenu. Le but de la base n'était pas de donner accès à la connaissance sur les objets (compte tenu de l'évolution de cette connaissance) comme dans $M A V I$, mais à une étape de cette connaissance, telle qu'elle nous est livrée. Le problème se posait donc de prévoir ou non des compléments pour faire communiquer les données de cette connaissance avec celles de la connaissance actuelle. On ne saurait trop insister sur le lien entre les données et leur source, et il ne faudrait pas abâtardir le projet par un mélange mal maîtrisé.

Salomon Reinach, insatisfait des « irrégularités de classement 》 de son ouvrage, signale qu'il ne pourra pas revenir par la suite sur l'ordre des sculptures, si on lui fournit de meilleures images, car « il faudrait refaire l'index, et c'est là une besogne à laquelle on ne se condamne pas deux fois ", nous dit-il. Nous mesurons dans ses propos l'utilité de l'informatique qui permet, sans déranger l'existant, de corriger les erreurs de stratégie. Néanmoins, il ne faut pas sous-estimer la difficulté qui sera rencontrée pour comprendre sans interprétation la nature de certaines informations : l'auteur nous prévient qu'une information occupant telle place dans la notice ne devra pas toujours être interprétée de la même façon. C'est le cas tout particulièrement pour les mentions de lieux, tantôt lieu de découverte, tantôt lieu de conservation. Salomon Reinach, conscient de ces difficultés, signale : « Naturellement, mon travail ne s'adresse pas à ceux qui, trouvant le nom de Berlin après le numéro d'ordre d'une statue, s'imagineraient qu'elle a été découverte sur les bords de la Sprée. » Il nous reste à espérer que toutes les ambiguïtés soient aussi facilement décelables. On sait d'autre part que toute interprétation demandée à la personne qui indexe les données est source d'erreurs et occasionne une grande perte de temps, ce qui nous a obligés à veiller à la répartition par champs de ces informations, et, dans le cas présent, à regrouper les données de lieux dans un même champ.

\section{Accès aux données}

Nous avons pris le problème au rebours de la plupart des néophytes dans le domaine des bases de données, qui commencent par « décrire » ou au mieux « classer » les données en fonction des corpus existants et recherchent ensuite un moyen de les rendre 
accessibles. Nous avons d'abord réfléchi sur l'appareil de communication de la base avec ses utilisateurs. Nous ne parlons pas que de l'interface, mais aussi, et peut-être encore plus, des modes d'accès aux données. Ces dernières sont de deux types : images et textes. Nous avons étudié la pertinence d'un double accès fondé sur ces deux natures d'information. Le nombre rend l'accès direct par l'image rédhibitoire, encore plus à l'écran que dans les volumes. Nous devions donc reprendre le principe de «l'index» sur des critères textuels. Il ne s'agit plus d'un livre qu'on peut feuilleter mais d'une série de petits coffres forts dont le contenu peut rester à jamais caché si le consultant ne connaît pas le code d'accès à chacun d'eux. De même que l'index du livre permet de ne pas avoir à feuilleter tous les volumes, les mots clé permettent de ne pas faire défiler des centaines d'écrans d'images. Ors, quand il s'agit d'un livre, les lecteurs sont habitués à se contenter de l'index existant : ils savent bien que leur propre expression sera vaine si le mot n'existe pas dans l'index. En revanche, l'informatique a donné l'illusion à ses utilisateurs qu'ils pouvaient exprimer leur requête à leur guise. C'est le mirage des systèmes en « langage naturel », des moteurs de recherche full text. Le résultat n'a rien de rigoureux, les données inutiles étant en fait moins lourdes de conséquence pour le chercheur que les données manquantes.

Nous devions réfléchir sur la pertinence de l'index du livre pour retrouver les sculptures, sur l'exclusivité ou non de ce système d'interrogation. Nous avons débattu du bien-fondé d'une indexation parallèle pour faciliter la recherche, compte tenu des habitudes actuelles des spécialistes dans le domaine concerné. De même, pour la création des champs de la base, nous devions trouver un compromis utile entre la structure (fluctuante qui plus est) des données de l'ouvrage et la structure de classement des données telle qu'elle existe dans la mentalité des chercheurs qui utiliseront la base. C'est pourquoi nous avons mené une investigation dans le domaine des bases concernant la sculpture grecque et romaine, voire dans un domaine plus large de l'archéologie. Force est de constater que les habitudes sont très diversifiées, non seulement en ce qui concerne la terminologie, même au sein d'une seule langue, mais également dans la répartition conceptuelle des données. Bien que l'interrogation ciblée par champs paraisse la voie la plus sûre, nous avons fait l'expérience, devant des formulaires d'interrogation dite « experte » de ne pas savoir dans quel champ chercher une donnée précise. D'autre part, toute adjonction d'un vocabulaire d'indexation, toute organisation nouvelle en thésaurus induirait une modification de connaissance qui risquerait de dénaturer les données primaires du livre. Aussi sommes-nous arrivés à la conclusion de respecter les termes et les catégories de l'ouvrage, et de palier leur caractère éventuellement inhabituel en conduisant l'utilisateur de la base vers les contenus parmi lesquels choisir (liste des termes existant dans la base pour chaque champ), sans le laisser se risquer dans des voies sans réponses.

En ce qui concerne l'interface, la combinaison de l'image avec sa notice doit être étudiée par rapport à la proposition par planches de plusieurs statues dans l'ouvrage. Ici, nous sommes tenus de respecter le format des données dans la mesure où la répartition par images et la répartition par sujets ne sont pas liées explicitement. L'informatique permet plusieurs modes de « sortie » à partir des mêmes données. Nous avons étudié plusieurs modes possibles et utiles, afin qu'ils respectent les divisions créées par l'auteur et qu'ils se conforment aux attentes des consultants. Contrairement aux bases des musées 
qui peuvent associer sans risque l'image de l'objet et la totalité de sa fiche d'indexation, nous ne pouvons associer à chaque image un des éléments majeurs de cette indexation : l'iconographie. En effet, plusieurs sujets peuvent concerner les statues d'une même page, sans que nous sachions comment les répartir parmi les sculptures. Ors, l'une des interrogations les plus fréquentes concerne l'iconographie. Il ne sera pas possible de conduire l'utilisateur jusqu'à l'image de la statue à partir de l'iconographie; l'utilisateur recevra l'image de la page entière où le thème recherché concerne au moins une statue de la page. A lui de bâtir son hypothèse d'attribution du thème à telle ou telle statue dont il pourra lire la notice soit directement (éventuellement par zoom) sur l'image de la page entière, soit, plus confortablement, dans une fiche textuelle liée à l'image de la statue, reprenant les indications de la notice qui, elles, sont attribuables sans ambiguïté. L'accès direct à l'image de la statue sera donc possible à partir des critères de la notice (matériau, typologie de dimension, lieux, voire inscriptions).

L'ouvrage présente l'avantage de ne pas contenir de longues descriptions, pavés difficiles à consulter sur l'écran. Il sera donc possible de créer des « pages écran » qui ne contraignent pas l'utilisateur à manipuler les « ascenseurs », opération qui empêche une vue synthétique des données.

Pour l'interface, plusieurs solutions de grandeur sont possibles : ou bien on définit à l'avance un nombre de pixels pour l'interface et les images, auquel cas les images sortiront sur l'écran dans une qualité prévisible; ou bien l'interface pourra s'adapter à la taille de chaque écran, ce qui nécessitera un bon module de calcul de conversion d'échelle de l'image pour éviter les « escaliers » particulièrement perceptibles lorsqu'il s'agit d'images au trait. L'avantage de cette dernière solution est de s'adapter à l'évolution des matériels de diffusion informatique sans avoir à développer à nouveau l'interface. Cela implique une bonne résolution pour faciliter le calcul des plus grands formats de consultation (voir infra).

Le projet concerne un corpus fini : il serait donc possible de le diffuser par disque. Mais il convient d'envisager les avantages qu'apporterait la diffusion en ligne dans le monde de la recherche. Il s'en suivra un ensemble d'adaptations nécessaires compte tenu de la présence de caractères grecs dans l'ouvrage. Les polices doivent être partagées entre la base et ses utilisateurs. D'autre part, seule une commission scientifique pourra décider de la nécessité de traduire les mots clé en anglais pour en faciliter l'accès international. Dans ce cas, la présence simultanée du terme d'origine en français et de sa traduction anglaise pourrait limiter le risque de déviation sémantique, tout en permettant d'afficher la liste par ordre alphabétique soit en français, soit en anglais.

\section{La saisie des données}

Nous avons expérimenté la numérisation des images. Les dessins au trait sur fond «blanc » ne doivent pas être perturbés par d'éventuels effets de transparence. La résolution de saisie a été étudiée par rapport à la visualisation sur l'écran. Le but n'est pas de scruter le style du dessinateur comme on pourrait le faire d'un artiste, mais d'identifier l'objet. Pour cela, étant donné la simplicité des dessins, il est inutile de consulter l'image d'une statue à la taille de l'écran. Il convenait également de trouver 
un compromis entre la vision entière de la page et la lecture des textes, si possible sans avoir à naviguer dans une image plus grande que l'écran. Il reste actuellement peu de consultations en 800 x 600 pixels, taille qui ne permet de déchiffrer les textes d'une page plein écran qu'à la condition d'appliquer un bon filtre de conversion d'échelle, quelle que soit la qualité de la numérisation. Nous avons constaté qu'une numérisation à 300 points par pouce supporte mieux la réduction qu'une numérisation à 150 points par pouce. Au pire (sur un écran de $600 \times 800$ points) une page numérisée à 300 points par pouce tiendra en hauteur à $25 \%$ de sa pleine résolution, en rognant en hauteur des parties blanches. Ce coefficient de conversion d'échelle est favorable, dans certains logiciels, à une meilleure lecture, du dessin comme du texte. Encore faudraitil alors figer la taille de l'image et de l'interface. Notons qu'il s'agit là du minimum valable. Monter jusqu'à la résolution de 300 points par pouce permettrait d'agrandir à l'écran un détail, fonction très utile pour déchiffrer certains chiffres dans les références bibliographiques, qui sont mal venus à l'impression. Cette fonction devrait rester exceptionnelle, mais elle semble nécessaire et justifie cette qualité de saisie. Bien que les capacités de stockage des données ne constituent plus actuellement une barrière à la haute résolution, il sera inutile d'utiliser les images «brutes de scan » : la compression permet de faire passer une page en noir et blanc du répertoire de 3,16 Mo à 834 Ko en compression Tif LZW, ou encore à 556 voire 200 Ko en compression Jpeg, tout en offrant une bonne lisibilité en pleine page dans l'écran et un excellent contrôle en pleine résolution par zoom. La légèreté du fichier permettra d'afficher plus rapidement l'image.

La note de l'auteur, dans l'introduction, concernant l'épaisseur des traits après les réductions des originaux, doit être prise en compte, surtout dans la mesure où les dessins risquent d'être observés à des agrandissements différents. Là encore, la qualité du filtre de réduction de l'image interviendra dans la grosseur du trait et dans le contraste du trait avec le fond. Nous retrouvons le problème, mentionné supra, du choix entre l'interface figée et l'interface adaptable à la taille de l'écran récepteur. Il reste possible de traiter les images en amont, mais leur nombre rend l'opération handicapante pour la rapidité de mise en œuvre de la base, sauf si le traitement est intégré à la numérisation, ce que permettent les bons scanners.

Nous avons mis au point une structure qui fait fonctionner les mises en relation de l'ouvrage, telles qu'elles apparaissent entre les images et les notices, les différentes données abrégées des notices et leur expression détaillée dans les index, entre les index et les images. Nous avons évité d'atomiser les données des notices en prévoyant les dangers que cela pourrait éventuellement présenter (voir supra l'exemple des lieux). La saisie nécessitera une grande attention car il faudra traduire l'absence de certaines données comme étant la mention d'une donnée. Ainsi, l'auteur signale « L'absence de toute indication autre que B. signifie petit bronze; quand il n'y a aucune indication de dimension ni de matière, il s'agit d'une statue de marbre, plus ou moins voisine de la grandeur naturelle. » Une aide à la saisie par listes de termes éclairés par des «bulles » d'information reprendra ces conventions pour les matériaux et les dimensions.

Des expériences devront être menées pour simuler le fonctionnement de la base dès la constitution d'un nombre très limité d'items, pour permettre une refonte rapide de la structure et du système de requête. Après avoir débattu de l'opportunité d'une 
saisie des données indépendante du système de la base (par exemple dans un tableur) ou d'une saisie directe dans le système de base de données, nous pensons que cette dernière solution serait à la fois plus rapide, plus aisée et entraînerait moins d'erreurs de ventilation des données d'un logiciel à l'autre. Il suffira de construire une interface adaptée au projet. Le choix du logiciel devra favoriser une mise en œuvre facile mais aussi supporter les milliers de sculptures du répertoire de Salomon Reinach et prévoir le nombre d'accès simultanés possibles. Il conviendra de dépasser le niveau des logiciels intégrés de la bureautique, quelles qu'en soient les possibilités dans des projets heuristiques. La pérennité de la base risque de dépendre de la pérennité du système choisi, bien qu'il soit possible de convertir les données d'un système à un autre. En dernier ressort, c'est l'implication humaine qui sera le meilleur garant de la survie de la base, implication de ses gestionnaires mais aussi des chercheurs qui devraient trouver là un outil dont la valeur est à la fois historique et synthétique, un outil qui dépasse l'éclatement par musées ou par nations du patrimoine de la statuaire grecque et romaine.

Enfin, notre implication dans la constitution du Musée achéménide virtuel et interactif (MAVI), sous la direction du professeur Pierre Briant au Collège de France, nous a permis d'approfondir notre expérience, tant dans le domaine des bases de données que dans celui de la mise en ligne, expérience que nous avons mise au service du projet Salomon Reinach. 